\title{
Erratum to: Almost automorphic mild solutions to some classes of nonautonomous higher-order differential equations
}

\section{Toka Diagana}

Received: 3 September 2011 / Accepted: 4 April 2012 / Published online: 4 June 2013

(C) Springer Science+Business Media New York 2013

\section{Erratum to: Semigroup Forum (2011) 82:455-477 \\ DOI 10.1007/s00233-010-9261-y}

In this Note we correct some errors that occurred in our paper [T. Diagana, Almost automorphic mild solutions to some classes of nonautonomous higher-order differential equations. Semigroup Forum. 82(3) (2011), 455-477].

I. It was recently found that Lemma 3.7 in Diagana [1], which is taken from the paper by Goldstein and N'Guérékata [2], contained an error. Indeed, the injection $B C^{1-\beta}\left(\mathbf{R}, \mathbf{X}_{\alpha}\right) \hookrightarrow B C(\mathbf{R}, \mathbf{X})$ as stated in both [1] and [2], is in fact not compact and that is crucial for the use of the Schauder fixed point to prove the existence of an almost automorphic solution to Eq. (3.1) appearing in [1]. The above-mentioned issue has been fixed in a recent Note by Goldstein and N'Guérékata [3]. The main objective of this paper is to correct Lemma 3.7 and slightly modify assumptions (H.4)-(H.5) to adapt Theorem 3.8 of [1] to this new setting.

A function $f \in B C(\mathbb{R}, \mathbb{X})$ is said to belong to $A A(\mathbb{X})$ [resp., $\left.A A_{u}(\mathbb{X})\right]$ if for every sequence of real numbers $\left(s_{n}^{\prime}\right)_{n \in \mathbb{N}}$ there exists a subsequence $\left(s_{n}\right)_{n \in \mathbb{N}}$ such that

$$
\lim _{n \rightarrow \infty} f\left(t+s_{n}\right)=g(t), \quad \lim _{n \rightarrow \infty} g\left(t-s_{n}\right)=f(t)
$$

pointwise on $\mathbb{R}$ [resp., uniformly on compacts of $\mathbb{R}]$.

Communicated by Jerome A. Goldstein.

The online version of the original article can be found under doi:10.1007/s00233-010-9261-y.

T. Diagana (凶)

Department of Mathematics, Howard University, Washington, DC 20059, USA

e-mail: tdiagana@howard.edu 
In contrast with [1], here the space $B C^{\gamma}\left(\mathbf{R}, \mathbf{X}_{\alpha}\right)$ will be viewed as a locally convex Fréchet space equipped with the following metric (see [3])

$$
\Delta(f, g)=\sum_{n=1}^{\infty} 2^{-n} \frac{\rho_{n}(f, g)}{1+\rho_{n}(f, g)}
$$

where, for $h=f-g$,

$$
\Delta_{n}(f, g)=\Delta_{n}(h, 0)=\|h\|_{C[-n, n]}+\gamma \cdot \sup \left\{\frac{\|h(t)-h(s)\|_{\alpha}}{|t-s|^{\gamma}}: t, s \in[-n, n], t \neq s\right\} \text {. }
$$

Let $\mathrm{GN}_{\gamma}\left(\mathbb{R}, \mathbb{X}_{\alpha}\right)$ be the locally convex Fréchet space $\left(B C^{\gamma}\left(\mathbb{R}, \mathbb{X}_{\alpha}\right), \Delta\right)$.

Lemma 3.7 in [1] should be replaced with

Lemma 0.1 The set $\mathrm{GN}_{1-\beta}\left(\mathbb{R}, \mathbb{X}_{\alpha}\right)$ is compactly contained in $\mathrm{GN}_{0}\left(\mathbb{R}, \mathbb{X}_{\alpha}\right)$, that is, the canonical injection id: $\mathrm{GN}_{1-\beta}\left(\mathbb{R}, \mathbb{X}_{\alpha}\right) \hookrightarrow \mathrm{GN}_{0}\left(\mathbb{R}, \mathbb{X}_{\alpha}\right)$ is compact, which yields

$$
\text { id }: \mathrm{GN}_{1-\beta}\left(\mathbb{R}, \mathbb{X}_{\alpha}\right) \cap A A_{u}\left(\mathbb{X}_{\alpha}\right) \hookrightarrow A A_{u}\left(\mathbb{X}_{\alpha}\right)
$$

is compact, too.

II. For the matrix $A_{l}(t)$ in Eq. (4.1) to be decomposed as $A_{l}(t)=K_{l}^{-1} J_{l}(t) K_{l}(t)$ as stated in [1], one has to suppose that each root $\rho_{k}^{l}(k=1,2, \ldots, n)$ of the polynomial $Q_{t}^{l}(\cdot)$ in page 457 is of multiplicity one.

III. Consider the following assumptions:

(h.4) $R(\omega, A(\cdot)) \in A A_{u}\left(B\left(\mathbb{X}_{\alpha}\right)\right)$.

(h.5) The function $F: \mathbb{R} \times \mathbb{X}_{\alpha} \mapsto \mathbb{X}$ is such that $t \mapsto F(t, u)$ belongs to $A A_{u}(\mathbb{X})$ for all $u \in \mathbb{X}_{\alpha}$. The function $u \mapsto F(t, u)$ is uniformly continuous on any bounded subset $K$ of $\mathbb{X}$ for each $t \in \mathbb{R}$. Finally,

$$
\|F(t, u)\|_{\infty} \leq \mathcal{M}\left(\|u\|_{\alpha, \infty}\right),
$$

where $\mathcal{M}: \mathbb{R}^{+} \mapsto \mathbb{R}^{+}$is a continuous, monotone increasing function satisfying

$$
\lim _{r \rightarrow \infty} \frac{\mathcal{M}(r)}{r}=0 .
$$

In view of the above, Theorem 3.8 in [1] should be replaced with

Theorem 0.2 Suppose assumptions (H.1)-(H.2)-(H.3)-(h.4)-(h.5) hold, then the nonautonomous differential equation (3.1) has a mild solution which belongs to $A A_{u}\left(\mathbb{X}_{\alpha}\right)$.

\section{References}

1. Diagana, T.: Almost automorphic mild solutions to some classes of nonautonomous higher-order differential equations. Semigroup Forum 82(3), 455-477 (2011)

2. Goldstein, J.A., N'Guérékata, G.M.: Almost automorphic solutions of semilinear evolution equations. Proc. Am. Math. Soc. 133(8), 2401-2408 (2005)

3. Goldstein, J.A., N'Guérékata, G.M.: Corrigendum on "Almost automorphic solutions of semilinear evolution equations". Proc. Am. Math. Soc. 140, 1111-1112 (2012) 